\title{
Barrier destruction and Lagrangian predictability at depth in a meandering jet
}

\author{
G.-C. Yuan ${ }^{\mathrm{a}, \mathrm{b}, *}$, L.J. Pratt ${ }^{\mathrm{b}}$, C.K.R.T. Jones ${ }^{\mathrm{a}}$ \\ ${ }^{a}$ Division of Applied Mathematics, Brown University, Providence, RI 02912, USA \\ ${ }^{\mathrm{b}}$ Woods Hole Oceanographic Institution, Woods Hole, Massachusetts, Woods Hole, MA 02543, USA
}

Received 12 December 2000; accepted 29 August 2001

\begin{abstract}
Numerical simulations of a jet with large amplitude meanders are used to explore chaotic advection processes and underlying geometry changes as functions of the ambient potential vorticity gradient $\beta$. Variations in $\beta$ in the $2 \mathrm{D}$ model qualitatively simulate changes in depth in $3 \mathrm{D}$, surface-intensified jets such as the Gulf Stream. As $\beta$ is reduced, corresponding to motion on increasingly deep isopycnal surfaces, a number of geometrical transitions take place in the flanges and across the core of the jet. The most important is a joining (or separatrix reconnection) of heteroclinic cat's eyes structures lying to the north and south of the jet core. The jet core acts as a barrier to transport, but this barrier is breached when the cat's eyes merge. The subsequent chaotic transport across the jet is demonstrated by calculations of effective invariant manifolds (EIMs) originating in hyperbolic regions to the north and south of the core. Destruction of the central barrier occurs as $\beta$ is lowered through a narrow window $W$ about $\beta=0$ and is marked by transitions form a meandering jet through a vortex street with no central meandering flow to a vortex street with a retrograde meander. Such small values of $\beta$ are deemed reasonable in view of measurements of low potential vorticity gradients in the deep Gulf Stream. The strength of the central barrier for $\beta$ outside $W$ is tested by varying $\beta$ about a mean value $\beta_{0}$ and detecting the minimum amplitude of fluctuation necessary for destruction of the barrier. It is found that the barrier is stronger for $\beta_{0}>0$, at least by this measure. A striking difference is that, for $\beta<0$, some disturbances may destroy the barrier without oscillating across $W$; whereas for $\beta>0$, destruction of the barrier may only occur when $\beta$ passes through $W$. Changes in underlying geometry also occur in the flanges of the jet and these changes alter the locations in which fluid is preferentially stirred and mixed.
\end{abstract}

\footnotetext{
* Corresponding author. Tel.: +1-401-863-2262; fax: +1-401-863-1355.

E-mail address: gcyuan@cfm.brown.edu (G.-C. Yuan).
} 
Float trajectories can be regular or irregular depending upon where the instrument is launched and this is demonstrated by plotting trajectories from inside and outside regions of chaotic advection. (c) 2002 Elsevier Science B.V. All rights reserved.

Keywords: Gulf Stream; Barotropic jet; Transport; Chaotic theory; Separatrix reconnection; Effective invariant manifold

\section{Introduction}

Studies of Lagrangian motion and chaotic advection in meandering jets (Behringer et al., 1991; Bower, 1991; Lozier and Bercovici, 1992; Samelson, 1992; Dutkiewicz et al., 1993; del-Castillo-Negrete and Morrison, 1993; Meyers, 1994; Pratt et al., 1995; Duan and Wiggins, 1996; Miller et al., 1997; Ngan and Shepherd, 1997; Rogerson et al., 1999) have dealt with model flows that typify the surface structure of ocean currents such as the Gulf Stream, the Kuroshio, the jets of the Circumpolar Current, and other surface-intensified, meandering flows. The central region or "core" of the jet in such models usually contains a strong potential vorticity gradient which acts as a barrier to cross-jet transport of fluid. Such transport is generally confined to the edges of the jet, near the critical lines of the meanders, where the fluid elements undergo the strong stretching and folding associated with Lagrangian chaos. The result is that passive tracers are preferentially stirred (and eventually mixed) along the edges, but not across the central core of the flow, a feature that seems to agree with property distributions in the shallow Gulf Stream (Bower et al., 1985). Shallow cross-jet transport thus appears to require a catastrophic event such as ring detachment.

The models referenced above say less about the mixing of fluid at depth. In the Gulf Stream, water mass properties are relatively homogeneous below the $27.0 \sigma_{\theta}$ surface (Bower et al., 1985), which extends about from $200 \mathrm{~m}$ depth to the north to $800 \mathrm{~m}$ depth to the south of the Stream. The overall cross-stream potential vorticity gradient below this depth diminishes (Bower and Lozier, 1994), reaching values that are, at certain depths and time, not significantly different from zero. Bower et al. (1985) also found that shallow floats tend to remain trapped in the Gulf Stream whereas deeper floats more frequently crossed the Stream "as if it was transparent". In addition, Cronin and Watts (1996) and Savidge and Bane (1999a,b) show that the deep Gulf Stream is dominated by strongly barotropic eddies that are spun up by baroclinic instability at shallower depths and which translate at the same speed as the shallow meanders. In an idealized view, the deep Gulf Stream is therefore thought to be less "jet-like" and more in the character of a vortex street. All of these observations are consistent with the presence of transport and mixing across the whole width of the flow.

A scenario describing how cross-jet exchange might take place at depth can be pieced together using ideas first mentioned by Bower and Rossby (1989) and later refined by Meyers (1994) and Pratt et al. (1995). First, refer to Fig. 1 showing a well-studied geometry thought to be typical of the surface structure of a meandering jet under idealized conditions. The jet flows eastward and contains a monochromatic meander that steadily propagates eastward at something less than the maximum fluid speed in the jet. In the frame of reference following the meander (the co-moving frame) the flow is steady; the corresponding streamlines are what appear in the figure. Fluid in the central core of the jet streams eastward through 


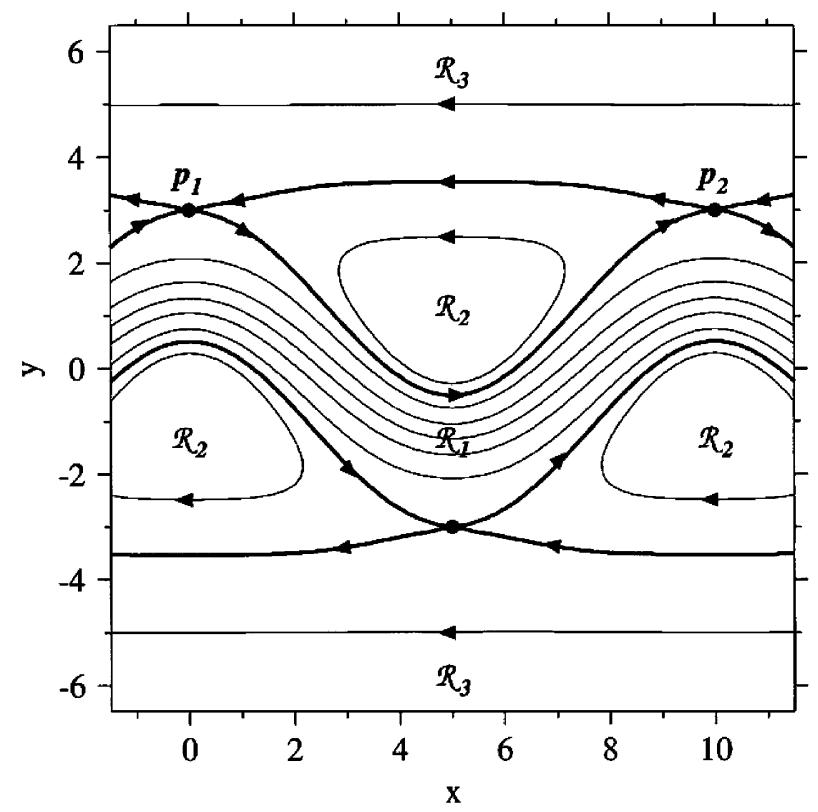

Fig. 1. Schematic of a meandering jet in the co-moving frame. Taken from Rogerson et al. (1999).

the frame while fluid far to the north and south appears to move westward. Between these streaming regions are lines of recirculations (or cat's eyes) surrounded by heteroclinic separatrices linked to hyperbolic stagnation points $p_{1}, p_{2}$, etc. The fluid trajectories in this flow are confined along streamlines and thus there is no fluid exchange between the regions of open and closed streamlines. However, the models cited above have established that small time dependent perturbations to the flow in the form of additional meanders or weak diffusion can cause the separatrices to break up and allow fluid exchange. It is also well-known that the fluid involved undergoes violent stretching and folding and that the corresponding parcel trajectories are chaotic. For weak perturbations the chaotic region extends around the edges of the former separatrices but does not extend into the core of the jet nor the centers of the cat's eyes. del-Castillo-Negrete and Morrison (1993) and Meyers (1994) show that sufficiently large perturbations can cause penetration into and across the jet core, but this behavior has only been observed in kinematic models or models using linear meander perturbations with amplitudes well beyond the range of dynamical consistency.

Next, consider the changes to the Fig. 1 geometry that might occur as one moves deeper in the water. We imagine the flow to be quasi-2D in the sense that parcel trajectories remain on isopycnal surfaces, but the flow can change on different isopycnal surfaces. The meander speed $c$ remains fixed but the jet speed $u$ weakens with increasing depth (or density) and therefore the hyperbolic stagnation points, which lie where $u(x, y)=c$ in the co-moving frame, migrate inwards towards the core of the flow. This suggests that the two rows of cat's eyes to the north and south of the core move closer to each other and perhaps merge, indicating destruction of the central behavior. Such a merger is called separatrix 
reconnection, and the aims of this paper are to establish conditions for reconnection in a dynamically consistent setting, to identify the associated geometrical transformations, and to explore implications for cross-stream transport and mixing.

A complete investigation of separatrix reconnection with changing depth suggests the use of a fully 3D model. However, we believe that a good deal of insight into the relevant issues can be gained through the careful use of a 2D model. This judgment is founded on the correspondence between moving to deeper levels in a 3D model and decreasing the value of $\beta$ in a 2D $\beta$-plane model. This analogy is corroborated by three features. First, decreasing $\beta$ in the $2 \mathrm{D}$ models causes the jet meanders to propagate more rapidly to the east. (The meanders are similar to Rossby waves which attempt to propagate westward but which are advected to the east by the jet itself. Decreasing $\beta$ diminishes the tendency to westward propagation.) The net effect is that the meanders propagate more rapidly relative to the background flow, bringing the meander cat's eyes closer to each other. Secondly, decreasing $\beta$ in the $2 \mathrm{D}$ model diminishes the overall potential vorticity change across the jet, the same effect that is observed at depth in the Gulf Stream. Finally, sufficiently low values of $\beta$ cause the 2D flow to become increasingly eddy dominated, a situation similar to what is observed.

Though self-consistent, the dynamics of the 2D model is different from the dynamics governing the quasi-2D flow on an isopycnal surface of a 3D flow. Nevertheless, it is anticipated that the 2D model will provide insight into the geometrical changes that can occur and the effort needed to break the central barrier to transport. In addition to providing information about stirring and mixing processes, the underlying geometry provides a template for understanding the Lagrangian predictability of the flow. As described above, regions of strong stretching and folding of fluid elements, leading to complicated and unpredictable Lagrangian motion, can exist in a field of relatively regular and predictable motion. Recognition of the geometry controlling the location of barriers and of regions of unpredictable motion can be an important consideration in the design of float and drifter experiments.

\section{Model}

The velocity fields used correspond to equilibrated, finite-amplitude states resulting from the instability of a Gaussian zonal jet. If $L^{*}$ and $U^{*}$ represent the half width and peak velocity in the undisturbed jet then length, velocity, and time scales for the problem can be chosen as $L^{*}, U^{*}$, and $L^{*} / U^{*}$. Using these scales for non-dimensionalization, the formal initial value problem is

$$
\begin{aligned}
& \frac{\partial q}{\partial t}+J(\psi, q)=\mu \nabla^{4} \psi, \\
& q=\nabla^{2} \psi+\beta y,
\end{aligned}
$$

and

$$
\psi(x, y, 0)=\Psi(y)+\varepsilon \mathrm{e}^{-y^{2}} \sin (k x),
$$

where $\Psi(y)=-\operatorname{erf}(y)+2 y / L_{\mathrm{D}}$. Note that Eqs. (2.1a) and (2.1b) is the barotropic potential vorticity equation with small lateral viscous damping and $q$ denotes the barotropic 
potential vorticity. In the analogy with quasi-2D motion on an isopycnal surface, we associate Eq. (2.1b) with the more general Ertel potential vorticity, approximated by Bower and Lozier (1994) as $\left(f_{0}-\partial u / \partial y\right) / H$ in their Gulf Stream measurements. In their analysis, the Coriolis parameter $f$ is approximated by its mean value $f_{0}$ (the variation of $f$ across the narrow flow being small compared to remaining terms) and the relative vorticity by the cross-stream gradient of along-axis velocity $-\partial u / \partial y$. The potential vorticity gradient at deeper levels is dominated by variations in the thickness $H$ between isopycnal levels. As depth increases, $H$ becomes more uniform and thus the potential vorticity gradient across the flow diminishes, in some cases to values that are not significantly different from zero. It is this effect that we associate with decreasing $\beta$ in the simulations described below.

Eq. (2.2) represents the initial zonal jet plus a small, meandering perturbation. Solutions are obtained numerically in a doubly periodic, $L_{\mathrm{D}}$ by $L_{\mathrm{D}}$ domain using a pseudospectral numerical code developed by Flierl et al. (1987) and refined by Rogerson et al. (1999). The reader is referred to the latter for more details regarding the code itself. The solutions presented here are obtained using $\varepsilon=0.02, \mu=10^{-3}$, and $L_{\mathrm{D}}=25.6$. The most important adjustable parameters in the problem are dimensionless beta $\left(\beta=\beta^{*} L^{*} / U^{*}\right)$ and the dimensionless initial wave number $k=L_{\mathrm{D}} k^{*}$. Flierl et al. (1987) made numerical runs over a grid of $(k, \beta)$ values and mapped out the finite-amplitude states that developed after saturation of the initial instability. Rogerson et al. (1999) further analyzed the subspace in which the evolution resulted in the formation of a meandering state with nearly steady meander speed and amplitude. The setting $(k, \beta)=\left(6 \pi / L_{\mathrm{D}}, 0.103\right)$ results in such a meander and this serves as the starting point for the numerical runs described here. We will discuss the equilibrated states that result by maintaining this $k$ and gradually reducing the value of $\beta$. In each case, the initial flow will evolve to an equilibrated state that is dominated by a meander or vortex street pattern (whose wave number equals the initial wave number) which persists over many time periods of the dominant pattern before it undergoes a secondary instability. Our analysis of transport and stirring in the flow is carried out over this finite period of persistence and nearly constant translation of the finite-amplitude pattern. In the co-moving frame the time dependence is weak and is dominated by one or two periods. ${ }^{1}$

\section{Separatrix reconnection}

Fig. 2(a) shows a snapshot of the co-moving frame stream function for the equilibrated flow corresponding to $\beta=0.103$. The central region of eastward streaming flow with vortical motion to the south of meander crests and to the north of the troughs is suggestive of the heteroclinic "cat's eye" geometry discussed above. The meanders propagate eastward at a nearly steady speed $c \approx 0.115$ and persist over the time interval $200<t<400$ with little change in amplitude. Fig. 3(a) shows the distribution of the potential vorticity contours for the same time slice of the flow. Notice that the gradient is large in the jet core, suggesting a barrier. As $\beta$ is decreased, $c$ increases but the central barrier persists. However, further reduction of $\beta$ to 0.01 leads to a flow field in which the central band of the streaming flow (or "core") is absent. As shown in Fig. 2(b), which displays the case $\beta=0$, stream function

\footnotetext{
${ }^{1}$ Rogerson et al. (1999) present frequency spectra for some of the $\beta>0$ case.
} 
(a)

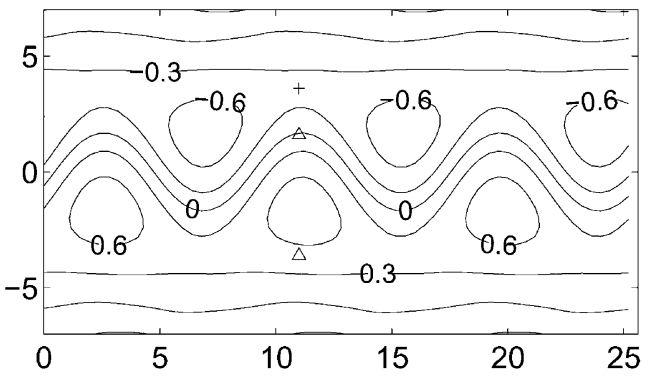

(b)

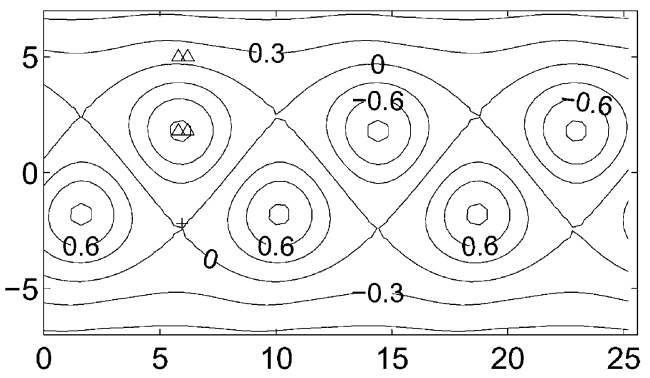

(c)

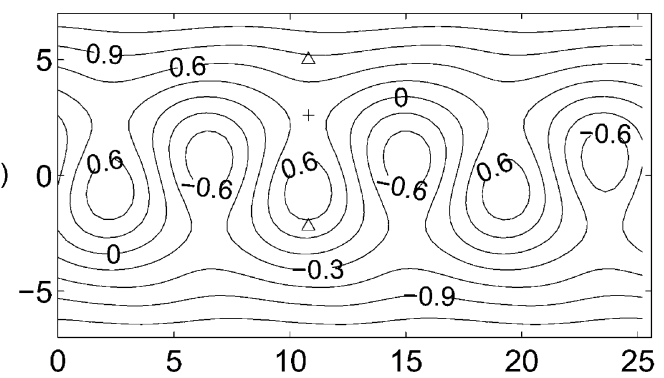

Fig. 2. Snapshot of streamline contours in the co-moving frame. (a) $\beta=0.103$; (b) $\beta=0$; (c) $\beta=-0.05$. The ' + ' and ' $\triangle$ ' mark the initial conditions of the trajectories in Fig. 9.

contours bounding the vortical motions now form a braided pattern suggestive of the separatrix connection discussed by del-Castillo-Negrete and Morrison (1993). The corresponding potential vorticity distribution, displayed in Fig. 3(b), shows a vortex street pattern. The potential vorticity gradient is significant only in the interior of the eddies. This "separatrix reconnection" pattern in contour plots of stream function and potential vorticity occurs only for a narrow window $W$ estimated by $-0.01<\beta<0.01$. When $\beta$ is further decreased, we observe a new geometry as shown in Fig. 2(c) for $\beta=-0.05$. In this case, the eddies are isolated from one another by a band of fluid that meanders through them (westward in the comoving frame). A homoclinic geometry is suggested by the streamlines separating the eddies from the meandering band. The potential vorticity distribution (Fig. 3(c)) suggests a vortex street pattern, now with a potential vorticity gradient between the eddies. 

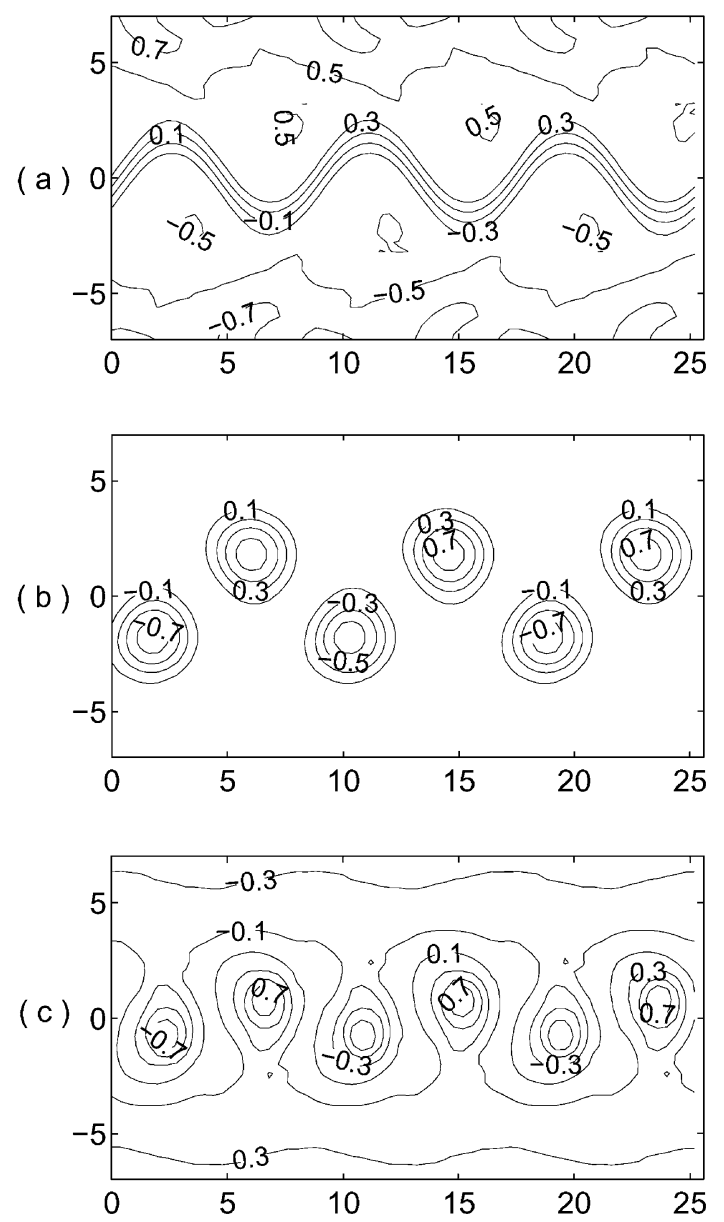

Fig. 3. The potential vorticity distribution corresponding to Fig. 2. (a) $\beta=0.103$; (b) $\beta=0$; (c) $\beta=-0.05$.

Although the band of strong potential vorticity gradient in the core persists as $\beta$ is lowered through positive values, some interesting transitions take place in the jet flanges. These will be detailed in the next section.

We also investigate the conditions for central barrier destructions for different basic jet profiles. In particular, for both a symmetrical and asymmetrical Bickley jet, we observe geometry changes similar to those discussed above. As summarized in Table 1, the window $W$ in which reconnection occurs is quite narrow and is centered at $\beta=0$, even in the asymmetrical case.

$$
\begin{cases}-\operatorname{erf}(2 y) / 2+3 y / 2 L_{\mathrm{D}}, & \text { if } y>0 \\ -\operatorname{erf}(y)+3 y / 2 L_{\mathrm{D}}, & \text { if } y \leq 0\end{cases}
$$


Table 1

The location of the separatrix window for different initial jet profiles

\begin{tabular}{lll}
\hline Jet profile & $\Psi(y)$ & $W$ \\
\hline Flierl et al. (1987) & $-\operatorname{erf}(y)+2 y / L_{\mathrm{D}}$ & $-0.01<\beta<0.01$ \\
Bickley & $-\tanh (y)+2 y / L_{\mathrm{D}}$ & $-0.005<\beta<0.005$ \\
Non-symmetric & $-\operatorname{erf}(2 y) / 2+3 y / 2 L_{\mathrm{D}}$, if $y>0$ & $-0.01<\beta<0.01$ \\
& $-\operatorname{erf}(y)+3 y / 2 L_{\mathrm{D}}$, if $y \leq 0$ & \\
\hline
\end{tabular}

An effective method for assessing transport across active flow regions, such as the jets considered here, can be based on chaotic advection. In this theory, the tangling of stable and unstable manifolds of certain distinguished stagnation, or periodic, points in the flow field creates a zone in which parcel motion is chaotic. Another view of this same scenario is that the stable and unstable manifolds delineate regions of distinguished dynamic fate and their tangling marks off regions of fluid that switch from one flow regime to another, so-called fluid exchange. This technique has led to the theory of lobe dynamics and chaotic transport (Wiggins, 1992).

The use of stable and unstable manifolds as orchestrating fluid exchange, in the way indicated above, requires the presence of a saddle fixed or periodic point in the flow field. Under consideration here are flows that exhibit sufficiently complex time dependence in which such fixed or periodic points cannot be expected to occur. However, the key local properties of stretching and compressing in complementary directions, called hyperbolicity, can still be present to an extent that affords an analogous theory. Lobe dynamics was extended to the aperiodic case (Malhotra and Wiggins, 1998); however, the flow fields under consideration here persist over only finite spans of time and theories based on the usual asymptotic conditions are not directly applicable.

The key observation is that, although no distinguished hyperbolic fixed or periodic points will be present, distinguished invariant manifolds akin to the stable and unstable manifolds of fixed or periodic points are present in even quite complicated flow fields. This theory was first developed in Miller et al. (1997) and Rogerson et al. (1999) and depends on isolating localized regions, rather than points, where there is strong hyperbolicity over the time interval of interest. Based on the dynamics in such a region, effective invariant manifolds (EIMs) can be generated in a fashion entirely analogous to the case where hyperbolic fixed points, or trajectories, are present. EIMs are then time slices of distinguished material surfaces. They are pinned by the hyperbolic regions and supply transport templates as in the periodic case. The hyperbolic regions are found near stagnation points of the frozen-time, Eulerian field, see Haller and Poje (1998). Such a region can be found in the flows of Fig. 2 near each intersection of stream function contours. Under appropriate conditions, the EIMs are then defined to within a certain measurable uncertainty (Haller and Poje, 1998).

The numerical computation of the EIMs proceeds as follows (see, Miller et al., 1997; Rogerson et al., 1999 for details). For a fixed time interval $t_{1} \leq t \leq t_{2}$, the unstable EIM is computed by evolving in forward time a segment, which at the initial time $t=t_{1}$ is located in the hyperbolic region and aligned approximately along the unstable direction. The stable EIM is computed similarly, but by evolving in backward time. 

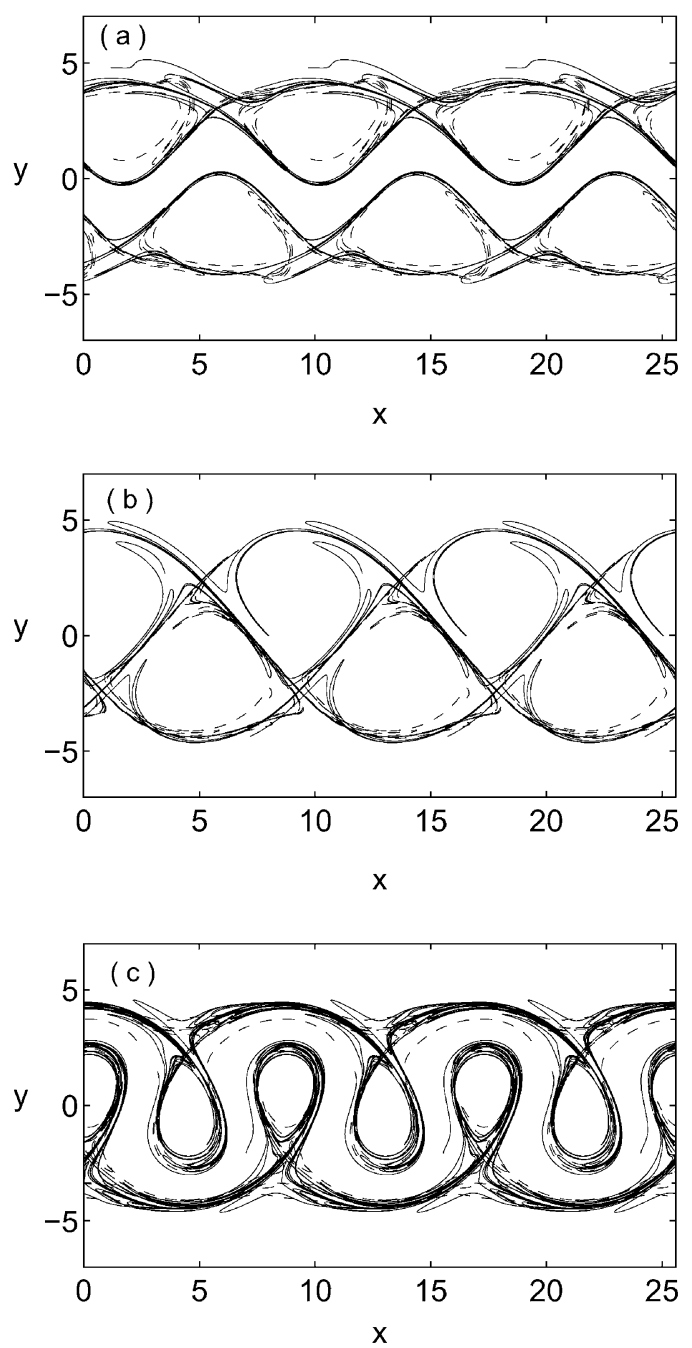

Fig. 4. Time slices of unstable (solid) and stable (dashed) EIMs. For easy visualization of transport, only the unstable EIMs originated from the north and the stable EIMs originated from the south are shown for $\beta=0$. (a) $\beta=0.103$; (b) $\beta=0$; (c) $\beta=-0.05$.

For $\beta=0.103$, we plot in Fig. 4(a) a time slice of stable (dashed) and unstable (solid) EIMs (for $200 \leq t \leq 400$ ). As mentioned above, fluid exchange is limited in the thin regions filled by the tangled EIMs. The lack of penetration of the EIMs into the jet core indicates that the core acts as a barrier for chaotic transport. In fact, one might define the barrier as the central region delineated by the inner envelopes of the regions of tangled EIMs.

At $\beta=0$ (see Fig. 4(b), for $150 \leq t \leq 300$ ), the unstable EIMs (solid curves) to the north clearly intersect with the stable EIMs (dashed curves) to the south, implying that 
cross-jet transport is achieved. In principle, one could calculate a volume flux associated with the transport by performing a lobe analysis along the line of Miller et al. (1997) and Rogerson et al. (1999). The lobes found between the interesting manifolds are quite thin and filamented, making analysis of the present case quite messy and difficult.

Fig. 4(c) displays the situation for $\beta=-0.05$ (for $100 \leq t \leq 300$ ), and indicates chaotic transport within homoclinic regions of quite large meridional extent. However, the EIMs to the north do not intersect with those to the south. Again, an impenetrable transport barrier is formed whose boundary is traced by the EIMs. In summary, cross-jet transport is observed only in a very narrow range of $\beta$ that approximately coincides with the separatrix reconnection window $W$ (estimated by inspecting the co-moving frame stream function contours).

\section{Transitions in the flanges of the jet}

The previous discussion emphasized the behavior of heteroclinic structures (cat's eyes) associated with the meander crests and troughs of the jet. Destruction of the central barrier occurs when two rows of cat's eyes straddling the jet core merge as $\beta$ is reduced below a threshold value $\beta \approx 0.01$. Interestingly, a careful examination of the flow field reveals that the cat's eyes themselves undergo significant changes just before this threshold is reached. As $\beta$ is lowered below the value 0.05 the cat's eyes merge with a band of secondary vortex structures (occupying a larger area than the cat's eyes) that move inwards towards the jet axis. This merger produces a relatively wide band of active chaotic advection on either side of the jet. As $\beta$ is lowered further, the motion in these bands reorganizes, producing new rows of cat's eyes. It is the reconnection of these new structures that destroys the central barrier. These transitions have implications for stirring along the flanges of the jet and are therefore worth detailing.

The above scenario can be followed in Fig. 5(a)-(c) showing potential vorticity fields for the cases $\beta=0.05,0.04$, and 0.02 . For $\beta=0.05$ the cat's eyes are indicated by the closed $q$ contours aligned along $|y| \approx 2.5$ in Fig. 5(a). Secondary rows of closed $q$ contours can be seen about $|y|=7$. These vortical structures translate at a nearly steady retrograde speed $c \approx-0.15$. They are touched by yet another row of disturbances centered at slightly larger $|y|$ and characterized by small fragments of close $q$ contours. The secondary regions appear to translate westward at a slightly greater speed than that of the vortical structures. We have discovered no simple description of these two sets of touching disturbances and will simply refer to the region occupied by both as the surf zone, roughly $5<|y|<10$ in Fig. 5(a). For higher $\beta$, the surf zones are present at about the same location and are associated with retrograde propagation, but the vortical structures are less discernible.

As $\beta$ is reduced the surf zones move inward and connect with the primary cat's eyes (Fig. 5(b), $\beta=0.04$ ). Further reductions (Fig. 5(c), $\beta=0.02$ ) lead to a reorganization within the connected regions, resulting in the formation of a new set of coherent and nearly stationary (in the co-moving reference frame) cat's eyes. Destruction of the central barrier occurs at $\beta \approx 0.01$.

In order to better understand the Lagrangian dynamics involved with this transition, we attempt to compute the EIMs associated with the cat's eyes and the surf zone. However, this 
( a )

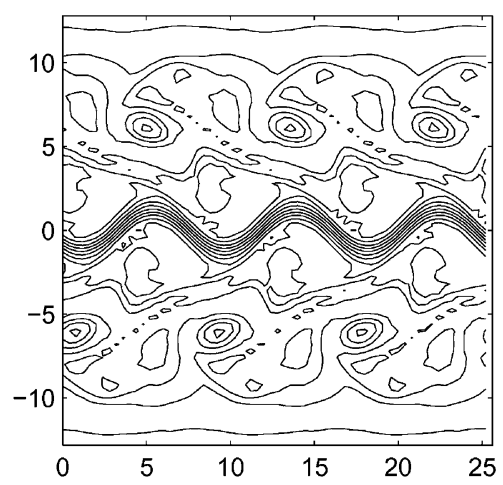

(b)

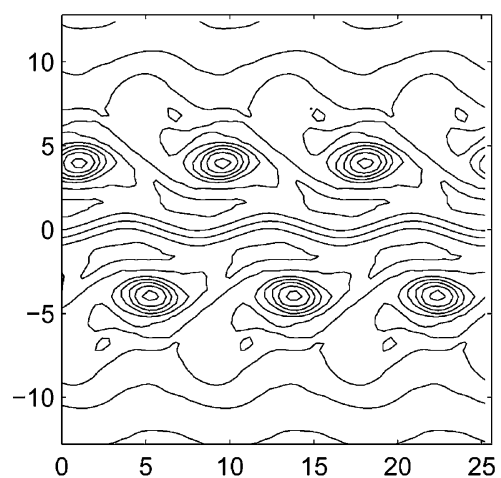

(c)

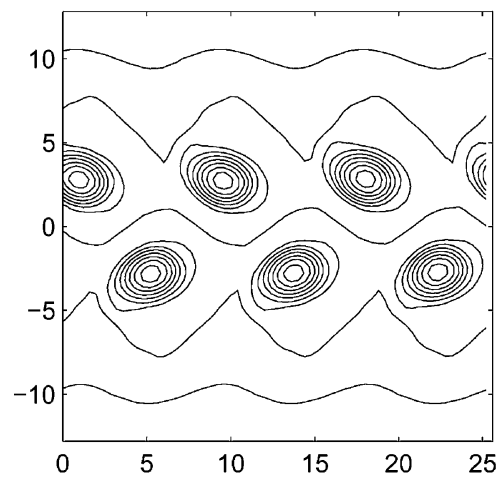

(d)

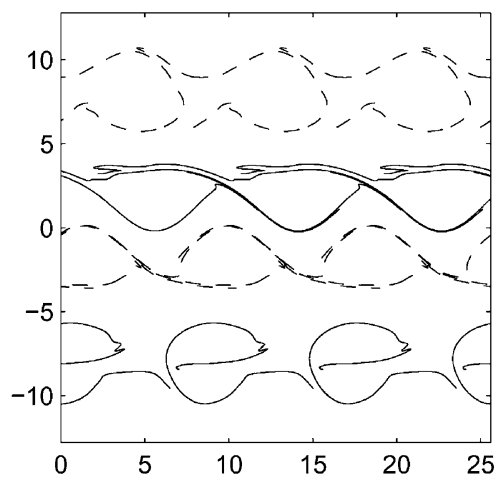

(e)

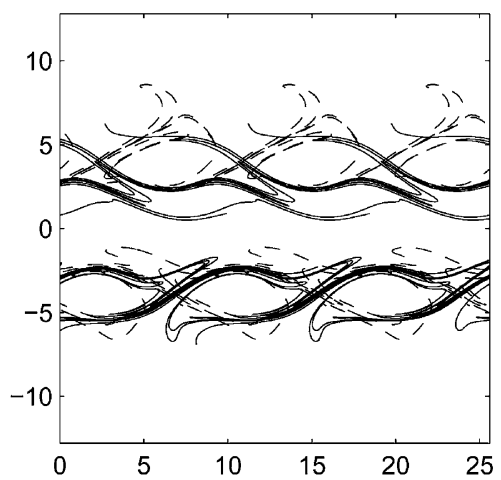

(f)

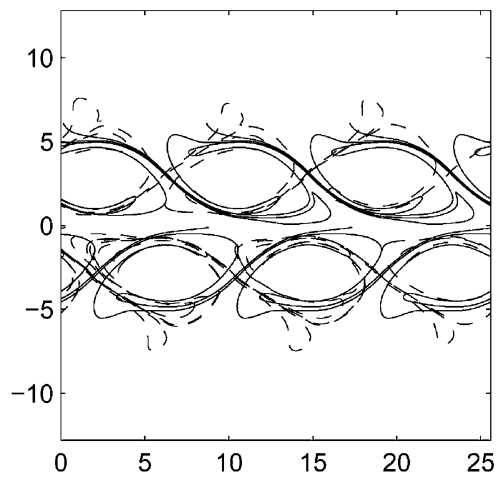

Fig. 5. Potential vorticity contours at $t=210$ of (a) $\beta=0.05$, (b) $\beta=0.04$, (c) $\beta=0.02$ and finite time stable (dashed) and unstable (solid) material curves for the same cases: (d) $\beta=0.05$, (e) $\beta=0.04$, (f) $\beta=0.02$.

task is more difficult than the previous cases as these regions strongly interact with each other and form a much wider, unified band and the hyperbolicity in each region is weakened. Nonetheless, the original cat's eyes and surf zone structures can be delineated by evolving carefully chosen material curves. The geometries of these material curves reflect different 


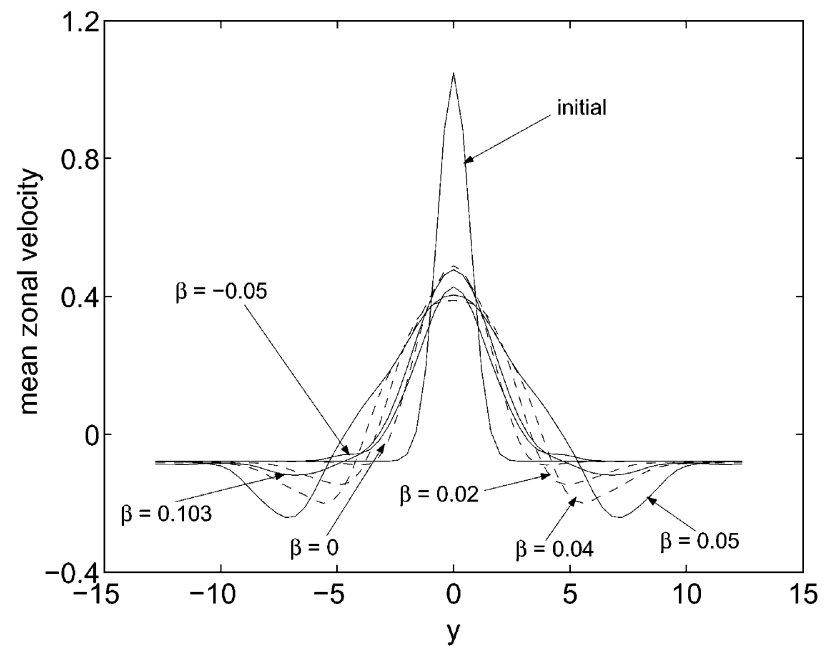

Fig. 6. The $x$-averaged zonal velocity $\langle u\rangle$ for various values of $\beta$. The initial zonal velocity (see Eq. (2.2)) is also plotted as a reference.

stages in this transition. These stable (dashed) and unstable (solid) material curves are shown in Fig. 5(d)-(f). For $\beta=0.05$ (Fig. 5(d)) the cat's eyes and surf zone appear as distinct bands with no connecting transport. For $\beta=0.04$ (Fig. 5(e)) the material curves from the two regions intersect, indicating transport within band occupying roughly $1 \leq|y| \leq$ 7. At $\beta=0.02$ (Fig. 5(f)), the material curves delineate well-defined heteroclinic cat's eyes.

The transitions described above are important in showing that destruction of the central jet barrier is associated not with reconnection of the original cat's eyes, but rather with reconnection of new cat's eyes. Although we do not entirely understand the existence of the surf zone and reasons for their inward motion, we have identified several illuminating features. For one thing, the surf zones are also bands of significant zonal flow rectification. As shown in Fig. 6, the mean ( $x$-averaged) zonal velocity $\langle u\rangle$ of the equilibrated flow contain side lobes of negative (retrograde) flow centered in the surf zone (e.g. at $|y|=7$ for $\beta=0.05)$. The existence of the side lobes is consistent with the relation between the acceleration of the mean flow and the divergence of the Reynolds stress $(\partial\langle u\rangle / \partial t=$ $-\partial\langle u v\rangle / \partial y)$, valid for our barotropic, doubly periodic flow and neglecting dissipation. For a linear perturbation $\operatorname{Re}\left[A \mathrm{e}^{\mathrm{i} k(x-c t)}\right]$ of the initial jet $U(y)$, this relation can be rewritten (Pedlosky, 1987) as

$$
\frac{\partial\langle u\rangle}{\partial t}=-k \operatorname{Im}(c)|A|^{2} \mathrm{e}^{2 k \operatorname{Im}(c) t} \frac{\beta-U^{\prime \prime}(y)}{|U-c|^{2}},
$$

suggesting that in the early stages of meander growth, the mean flow will experience deceleration where basic potential vorticity gradient $\beta-U^{\prime \prime}(y)$ is positive. The latter occurs about the jet axis (where $U^{\prime \prime}(y)<0$ ) and far from the axis (where $\left|U^{\prime \prime}(y)\right| \ll \beta$ ). It is in the latter region that the lobes of negative $\langle u\rangle$ are found. 
A second feature of importance is the westward propagating Rossby waves which are observed outside the surf zones for all positive $\beta$. These waves appear as wiggles in the $q$ contours near $|y|=11$ in Fig. 5(a). The waves cannot be forced directly by the eastward-propagating meanders of the jet core but could be excited by stochastic perturbations of the core about the meanders (Hogg, 1988). The jet side lobes are important in that they provide a setting in which these Rossby waves can have critical lines. For $\beta=0.05$, the waves propagate at speed $c \approx-0.14$ which is within the range of the mean velocity in the side lobes. Something like critical lines therefore exist in the side lobes and it is not surprising that a surf zone arises there. As Fig. 6 shows, the side lobes (and therefore the surf zones) move inwards as $\beta$ is decreased below the value 0.05 . We are unable to explain this inward movement.

\section{Quantifying the strength of the transport barrier}

In reality, an oceanic flow is never freely evolving but constantly disturbed by external forcings, which originate from various sources. A transport barrier that would have existed for the unforced flow might be destroyed by these additional disturbances. The sources of disturbances may have various frequencies, some are more effective than others. Therefore, it is interesting to test the strength of the central barrier against the disturbances stronger than those naturally occurring in the unforced flow and discriminate among different frequencies. Our model is far from being realistic. Nonetheless, we expect to discover some basic patterns that are similar to realistic situations.

First consider the situation where these disturbances are caused by forcing of a simplest form: the value of $\beta$ (in Eq. (2.1b)) is varied sinusoidally in time:

$$
\beta(t)=\beta_{0}+\delta \sin \omega t .
$$

To quantify the strength of the transport barriers, we fix $\omega$ whereas increasing $|\delta|$ gradually from zero until it reaches the threshold $\left|\delta_{c}\right|$ at which the stable EIMs to the south intersect with the unstable EIMs to the north, thus affording cross-jet transport. We thus view $\left|\delta_{c}\right|$ as a measure of the strength of the transport barrier.

To test how $\left|\delta_{c}\right|$ varies with the oscillation frequency $\omega$, we consider the two cases $\beta_{0}=-0.05$ (vortex street) and $\beta_{0}=0.05$ (meander). Table 2 summarizes the results for $\omega=0.04 \pi, 0.06 \pi, 0.08 \pi$, and $0.10 \pi$. Despite some uncertainty in the results for $\beta_{0}=0.05$, it is clear that $\left|\delta_{c}\right|$ increases with increasing $\omega$ in each case. At the same $\omega$, the values of $|\delta|$ required to destroy the $\beta_{0}=0.05$ barrier far exceed those for $\beta_{0}=-0.05$.

Table 2

Estimated $\left|\delta_{c}\right|$ for different $\omega$

\begin{tabular}{lllrr}
\hline$\delta_{c} \mid$ & $\omega$ & & \\
\cline { 2 - 5 } & $0.04 \pi$ & $0.06 \pi$ & $0.08 \pi$ & $0.10 \pi$ \\
\hline$\beta_{0}=-0.05$ & 0.03 & 0.05 & 0.06 & 0.13 \\
$\beta_{0}=0.05$ & $0.08 \pm 0.02$ & $0.15 \pm 0.04$ & $>0.30$ & $>0.30$ \\
\hline
\end{tabular}



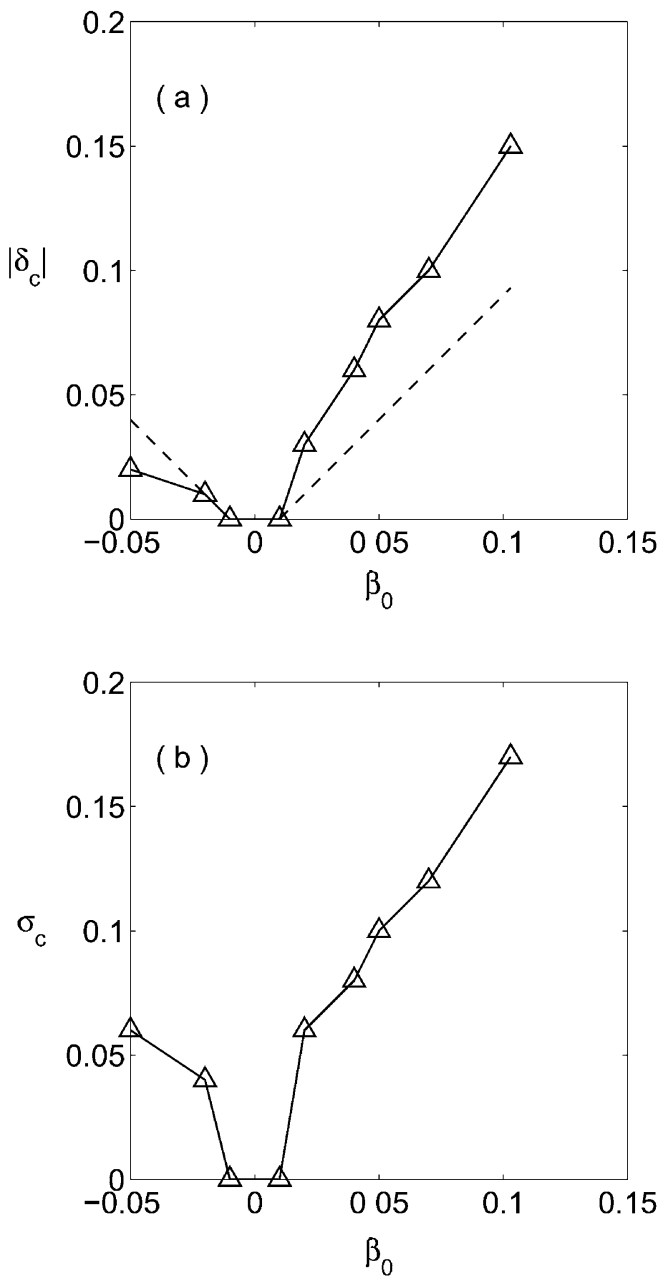

Fig. 7. The solid curve represents the estimated threshold amplitude of disturbance $\left|\delta_{c}\right|$ vs. $\beta_{0}$. Values marked with ' $\triangle$ ' are obtained through computations. The dashed curve is a plotted as a reference indicating the value of $|\delta|$ for $\beta(t)$ to marginally touch $W$. (b) Similar to (a) for $\sigma_{c}$ vs. $\beta_{0}$.

Within the above tested frequencies, $\omega=0.04 \pi$ is optimal for destruction of the transport barrier. It is possible that slower frequencies may lead to even smaller $\left|\delta_{c}\right|$. However, since only finite time (typically 200 time units) velocity fields are available for analysis, $\omega=$ $0.04 \pi$ is close to the slowest frequency that can be analyzed reliably. This choice allows $\beta(t)$ to oscillate about four cycles. Fig. 7(a) shows a plot of $\left|\delta_{c}\right|$ versus $\beta_{0}$ (the solid curve). It is obvious that $\left|\delta_{c}\right|$ increases as $\beta_{0}$ moves away from $W(-0.01 \leq \beta \leq 0.01)$. The dashed curve is plotted as reference and indicates the value of $|\delta|$ for $\beta(t)$ to marginally touch $W$. For $\beta_{0}>0,\left|\delta_{c}\right|$ is above the reference curve, indicating cross-jet transport can be achieved only if the disturbances are large enough to pass through $W$. On the other hand, for $\beta_{0}<0$, 
$\left|\delta_{c}\right|$ is below the reference curve, indicating cross-jet transport can be achieved without reaching the window $W$.

The above results suggest a striking difference between positive and negative $\beta$. In Section 3, we have shown that, for the unforced flow, transport can be achieved only in the separatrix reconnection window $W$. Thus it is unexpected that, for $\beta<0$, some disturbances $(\omega=0.04 \pi)$ may destroy the barrier without oscillating across $W$. This suggests that transport is enhanced by these disturbances in a non-trivial way. Although these "effective" disturbances are observed at special frequencies, these frequencies are likely to be contained in a broadband spectrum that the total disturbances may have. In contrast, such interesting enhancement does not occur for $\beta>0$, in which case, the barrier may be destroyed only when $\beta$ passes through $W$. These results suggest that small amplitude, broadband disturbances will likely cause transport for $\beta<0$ but not for $\beta>0$.

To further test the robustness of the above results, we replace Eq. (5.1) by

$$
\beta(t)=\beta_{0}+\sigma \eta(t)
$$

where $\sigma \eta(t)$ is a Gaussian white noise with standard deviation $\sigma$. Like in the previous cases, we increase $\sigma$ gradually from zero until it reaches the threshold $\sigma_{c}$ at which the transport barrier is destroyed. In Fig. 7(b), we plot $\sigma_{c}$ versus $\beta_{0}$. Again, we find it easier to destroy the transport barrier for $\beta_{0}<0$ than for $\beta_{0}>0$. Compare Fig. 7(a) with (b), we find $\sigma_{c}>\left|\delta_{c}\right|$ for all $\beta_{0}$. This is in agreement with the earlier result that the frequency $\omega=0.04 \pi$ is nearly optimal for creating cross-jet transport.

Finally it is worthwhile to point out that $\sigma_{c}$ defined above is a random number and fluctuates around its expected value. This is because each realization of $\eta(t)$ yields a new sequence of $\beta(t)$ (Eq. (5.2)), and thus a new velocity field, even when $\beta_{0}$ and $\sigma$ are both fixed. This randomness adds to the uncertainty of determining $\sigma_{c}$.

\section{Lagrangian unpredictability}

Chaos is generally associated with initial condition sensitivity caused by exponential divergence with time of nearby trajectories. Although the limitation of finite time in the present model does not allow chaos to be identified formally, we expect that certain regions of the flow field will exhibit very irregular Lagrangian motion and initial condition sensitivity.

An efficient technique for quantifying initial condition sensitivity is the patchiness plot first introduced by Malhotra et al. (1998) then developed by Poje et al. (1999). In essence, one computes the average of certain physical quantity $Q$ along the parcel trajectory starting from an initial condition $(x, y)$. Denote this average by $\bar{Q}(x, y)$. This computation is done for every point $\left(x_{i}, y_{j}\right)$ from a rectangular grid. If two trajectories remain close for most of the time, then the averages corresponding to these trajectories are almost identical. On the other hand, if for two nearby initial conditions $\left(x_{i}, y_{j}\right)$ and $\left(x_{i^{\prime}}, y_{j^{\prime}}\right)$, the difference $\left|\bar{Q}\left(x_{i}, y_{j}\right)-\bar{Q}\left(x_{i^{\prime}}, y_{j^{\prime}}\right)\right|$ is large, then the two trajectories must diverge from each other. The patchiness plot is the plot of the field $\bar{Q}$ versus the initial conditions $\left(x_{i}\right.$, $y_{j}$ ), and it serves as an aid for identifying regions with initial condition sensitivity, characterized by large magnitude of $|\nabla \bar{Q}|$. In Fig. 8(a)-(f), we show the patchiness plots for 
(a)
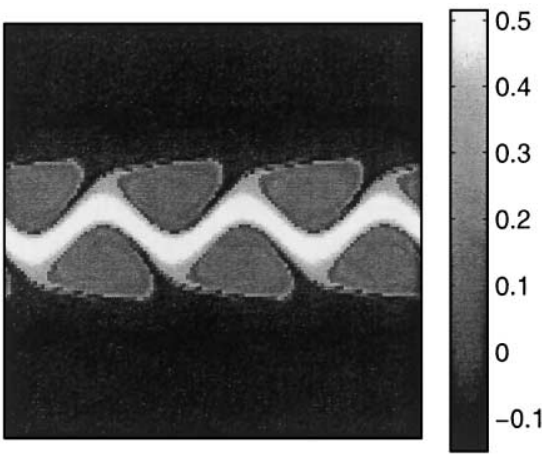

(c)
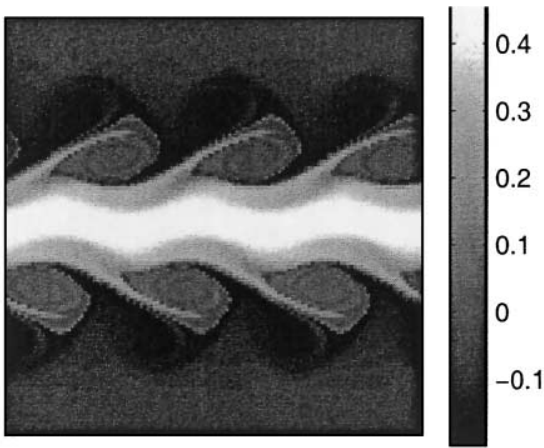

(e)
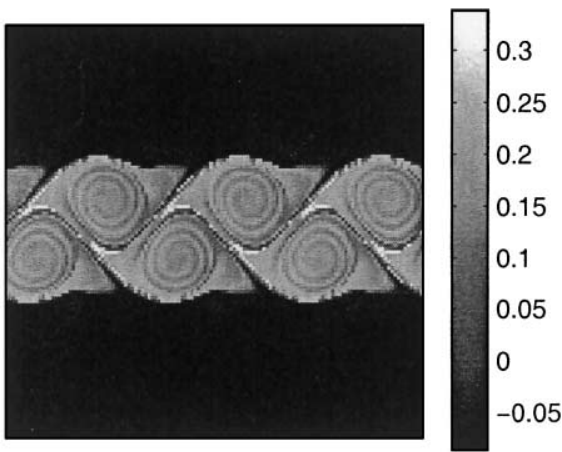

(d)
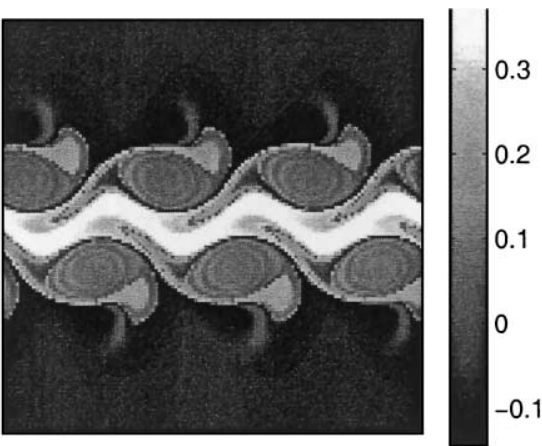

(b)
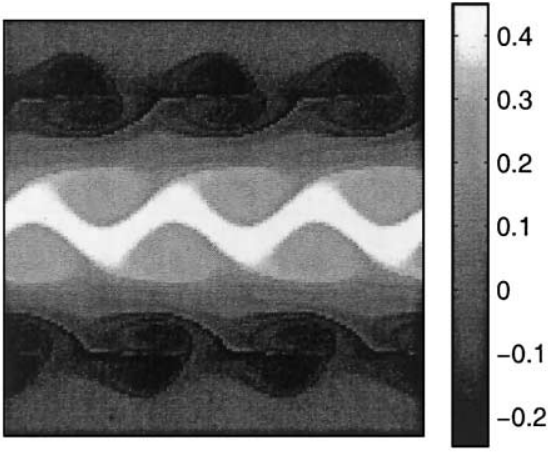

0.2

0.1
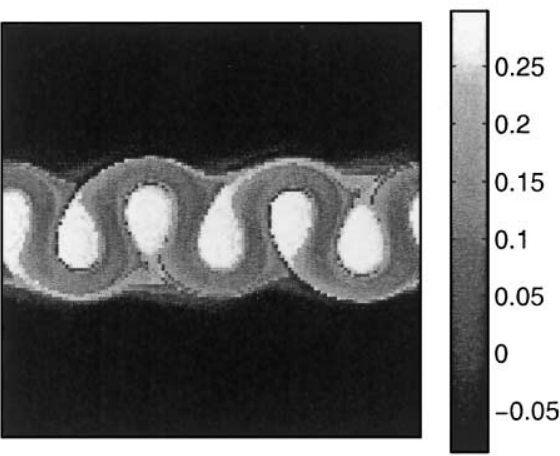

Fig. 8. The patchiness plots (see Section 6 for detail). (a) $\beta=0.103$; (b) $\beta=0.05$; (c) $\beta=0.04$; (d) $\beta=0.02$; (e) $\beta=0$; (f) $\beta=-0.05$.

$\beta=0.103,0.05,0.04,0.02,0$, and -0.05 . We choose the quantity $Q$ naturally to be the zonal velocity, thus $\bar{Q}$ is equal to the zonal distance travelled by each fluid parcel divided by the length of the time interval. It can be seen that the regions of "Lagrangian unpredictability" are occupied by trajectories that pass through areas of strong hyperbolicity and roughly correspond to the areas mapped out by the tangled EIMs in Figs. 4 and 5. Other regions 
such as the jet core, the interiors of the vortex street eddies, and the Rossby wave dominated far fields show up with relative initial condition insensitivity. ${ }^{2}$

Advanced knowledge of the geometry and distribution of both regular and irregular regions is helpful in formulating strategies for launching Lagrangian instruments. If the intension is that the instrument remains in a certain feature, such as the jet core or an eddy, then the launch site should lie well in the interior of such features and away from the tangled manifolds. On the other hand, studies of mixing may benefit from launch sites located in the irregular regions.

Our study suggests that significant changes in the distribution and geometry of irregular regions may occur with depth. Near the surface of our model flow, irregular regions occur around the edges of the cat's eyes associated with the eastward-propagating meanders, as shown in part (a) of Figs. 4 and 8. As $\beta$ is reduced (depth increases) secondary regions of irregularity may emerge, one example being the "surf zone" $(\beta=0.05)$ shown in Figs. 5(a) and (d), and 8(b). For this flow, an observer moving northward or southward from the jet core will encounter a regular region (the core), an irregular region in which regular pools are embedded (the cat's eyes), the regular band lying between the surf zone and the cat's eyes, the irregular surf zone itself, and finally the regular Rossby wave far field. Further reductions in $\beta$ lead to mergers of the cat's eyes and surf zones $(\beta=0.04$, Figs. 5(b) and (e), and $8(\mathrm{c}))$ resulting in the formation of a new set of cat's eyes $(\beta=0.02$, Figs. 5(c) and (f), and 8(d)). To this point, the irregular Lagrangian motion has been confined to the edges of the jet and has not penetrated the central core itself. However, reduction to $\beta \approx 0.01$ leads to destruction of the central barrier and formation of a vortex street across which the irregular motion can occur. The irregular region now has a braided geometry while regular regions consist of the vortices within the braids and the far field Figs. 4(b) and 8(e). A further slight reduction in $\beta$ leads to reformation of the central barrier and confinement of the irregular motion to homoclinic loops on either side of this barrier (Figs. 4(c) and 8(f)).

Characteristic regular and irregular motion in some of the regions just mentioned is shown in Fig. 9, which contains examples of parcel trajectories for flows with $\beta=0.103,0,-0.05$. The initial positions of trajectories in irregular regions are marked ' + ', while those in regular regions are marked ' $\triangle$ '. The initial positions are also marked in the corresponding Fig. 2 stream function maps. Trajectories originating at a ' + ' do indeed appear more irregular that those originating at a ' $\triangle$ ', the latter being nearly periodic. In some cases, pairs of trajectories have been initialized close together in order to observe initial condition sensitivity. The pair beginning near the ' + ' in Fig. 9(b), stays together for a brief period but rapidly diverge thereafter. Note that the northern member of the pair crosses the vortex street and remains on the north side of the street, a good example of cross-street transport. The two ' $\triangle$ ' pairs originating in the same figure remain together.

Ocean floats and drifters are not ordinarily launched in pairs, making it difficult to evaluate separation rates. However, promising directions have recently been suggested in Lacasce

\footnotetext{
${ }^{2}$ The spirals inside the eddies are rather curious. Similar patterns also appear in Poje et al. (1999) for barotropic turbulence, but the authors did not discuss the origin of this pattern. We find the underlying mechanism accounting for the robustness of this spiral pattern is the combination of two factors. First, parcels inside the eddies rotate around the eddy centers. Secondly, the locations of the eddies are not fixed but translated in time (in our case, we mean in the co-moving reference frame).
} 

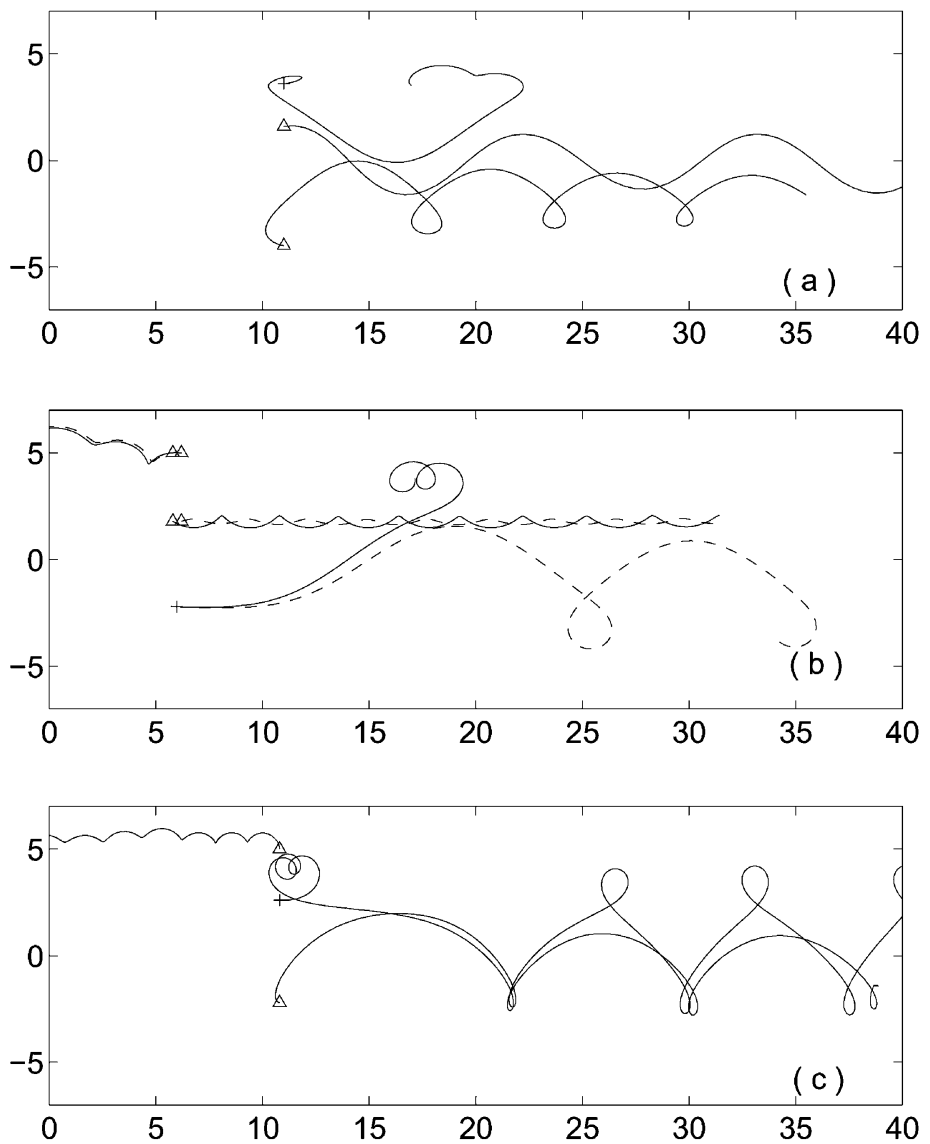

Fig. 9. Sample Lagrangian trajectories (in the stationary frame) for (a) $\beta=0.103$; (b) $\beta=0$; (c) $\beta=-0.05$. The initial positions are marked with ' + ' or ' $\Delta$ '.

et al. (2000). In particular, they have calculated the separation rates for a collection of instruments that have been launched in pairs or have passed close to one another, in several sections of the North Atlantic. They find that the mean square separations of most pairs initially grows non-linearly. This may be explained by the initial velocities of the instruments differing by a small constant, but an alternative explanation is that different pairs have different separation rates, and that certain pairs separate exponentially, as discussed in the above. In fact, Lacasce et al. (2000) do find that certain pairs separate much faster than others. Unfortunately, their analysis is limited by the facts that the time series are very short and that the data are contaminated by large noise. Therefore, they are not able to conclude whether there are pairs having exponential separation rates. They are currently working on data collected from the Gulf of Mexico, which have better quality. It is expected that more conclusive results will be obtained. 


\section{Conclusions and discussions}

By decreasing the value of $\beta$ in a model of a barotropic jet we have simulated the qualitative changes expected to occur with increasing depth (or density) in certain surfaceintensified, ocean jets. These changes include increased eastward meander propagation speed relative to the background eastward fluid velocity, decreased potential vorticity difference across the jet, and emergence of a flow with strong eddy motions and a weaker jet character. We decrease $\beta$ down to slightly negative values, deeming the latter consistent with observed values near zero in the Gulf Stream. Within this range, destruction of the central potential vorticity barrier and consequent cross-flow transport is observed only in the narrow window $W$ corresponding approximately to $-0.01<\beta<0.01$. Similar windows are found for jets of different shapes. Within $W$, the stream function contours in the co-moving reference frame take on a braided pattern that suggests separatrix reconnection. The corresponding flow has the character of a vortex street and transport across the street is demonstrated by the intersecting unstable and stable EIMs associated with the hyperbolic regions lying to the north and south of the street.

Motivated by the fact that an oceanic flow is disturbed by many external forcings, we test the strength of a transport barrier by introducing disturbances in our model. Although transport is not observed for the cases $\beta>0.01$ (a meandering jet with a high potential vorticity gradient core) and $\beta<-0.01$ (a vortex street with a continuous band of high potential vorticity gradient meandering between the vortices), transport can be forced in these cases by varying the value of $\beta$. However, the effect of this forcing is asymmetric with respect to $\beta$. For $\beta$-values of equal magnitude but opposite sign, the fluctuation required to destroy the transport barrier is larger for the positive $\beta$ than for the negative one. Within the fluctuation frequencies tested, the lowest of which permits four cycles over the finite duration of the solution, the barrier is most easily penetrated at low frequency. It is striking that, for $\beta<0$, some disturbances may destroy the barrier without oscillating across $W$; whereas for $\beta>0$, destruction of the barrier may only occur when $\beta$ passes through $W$. We also test the forcing due to a white noise disturbance of $\beta$. Again it is easier to destroy the transport barrier for negative $\beta$ than for positive $\beta$.

The central question to be addressed next concerns the depth (or density) range that the window $W$ corresponds to. Bower and Lozier (1994) estimations of monthly mean Gulf Stream potential vorticity gradient exhibit a good deal of temporal variability. For example, the potential vorticity gradient in a layer lying between 14.5 and $17.0{ }^{\circ} \mathrm{C}$ is significantly different from zero for all but several months of the 20 month period of observations. Note that this layer lies between $500 \pm 100 \mathrm{~m}$ depth at the south edge of the Gulf Stream and between $100 \pm 30 \mathrm{~m}$ at the north edge. For the 7.0-9.5 ${ }^{\circ} \mathrm{C}$ layer, which extends from $800 \pm 50 \mathrm{~m}$ on the south side to $350 \pm 50 \mathrm{~m}$ on the north side, the potential vorticity gradient is significantly different from zero for only a few of the 20 months. One might choose this and lower layers as candidates for separatrix reconnection and barrier destruction. However the full baroclinic model is clearly required to explore this issue in more depth.

It is not known whether the "homoclinic" vortex street found for $\beta<0.01$ is relevant to the deep Gulf Stream. It is certainly possible to find potential vorticity profiles that exhibit negative gradient across in the whole flow in the measurements of Bower and Lozier (1994). 
Quoted error bars for these cases indicate that the apparently negative gradients are not significantly different from zero.

Although our model suggests a mechanism for fluid exchange across a jet, it does not explain how fluid from afar can participate in this exchange. One must appeal to some large scale process such as the "cooling spiral" (Spall, 1992) to bring fluid into contact with the region of chaotic advection. In Spall's model, surface fluid in the North Atlantic subtropical gyre circulates anticyclonically in a downwards spiral. The sinking is caused by cooling in the western boundary layer and its extension. Due to dynamics analogous to the $\beta$ spiral, trajectories in the sinking region are forced to cross shallower trajectories from south to north. The net effect is that deep fluid is increasingly directed northwards, towards the axis of the jet separating the subtropical and subpolar gyres.

An incidental result of this investigation has been the observation of transitions in the flange region of the jet as $\beta$ approaches $W$ from above. Just before $W$ is reached, the (prograde) cat's eyes associated with primary meander at high $\beta$ combine with a (retrograde) "surf zone" to form a new set of cat's eyes. This merger creates relatively wide bands of chaotic stirring in the flanges. As $\beta$ enters $W$ the new cat's eyes merge and the central barrier is broken in what we call separatrix reconnection. Although the particulars of the flange transitions are likely model dependent, the existence and importance of secondary modes (here the retrograde of Rossby waves) implies that the process leading to barrier destruction at depth may not be as simple as previously thought. For example, Pratt et al. (1995) suggested a picture in which the jet is dominated in all depths by a normal mode meander. At the surface, a central barrier exists and chaotic stirring is confined to cat's eyes centered around the critical latitudes of the meander, much as in part (a) of Figs. 2-4. As one descends to deeper levels (or denser isopycnals) the critical latitudes move inwards and eventually merge, causing the central barrier to be broached. Our findings should serve as a reminder that other disturbances, for example those associated with Rossby wave radiation, may emerge at deeper levels and may alter the chain of events.

Hyperbolic regions and their EIMs give an underlying geometry that provides templates for chaotic stirring and transport of fluid in the jet. This study has identified a range of underlying geometries that could arise as depth is varied. Such changes would be important in the design of float and drifter experiments. If the intent is for the instrument to remain in certain regions, such as the jet core or a vortex structure, then advance knowledge of the locations of barriers and regions of enhanced stirring would be valuable.

\section{Acknowledgements}

We thank Audrey Rogerson for providing her original numerical code. Chi-Wang Shu helped us improve our numerical methods. The Lagrangian analysis is conducted using Patrick Miller's software: VFTOOL. We also thank Steve Meacham, Sanjeeva Balasuriya and Susan Lozier for helpful discussions. Finally, the use of the patchiness plot technique was suggested to us by an anonymous reviewer, to whom we express our sincere gratitude. This research was funded by the Office of Naval Research Grant N00014-92-J-1481 and N10014-99-1-0258. 


\section{References}

Behringer, R.P., Meyers, S.D., Swinney, H.L., 1991. Chaos and mixing in a geostrophic flow. Phys. Fluids A 3, 1243-1249.

Bower, A.S., 1991. A simple kinematic mechanism for mixing fluid parcels across a meandering jet. J. Phys. Oceanogr. 21, 173-180.

Bower, A.S., Rossby, H.T., 1989. Evidence of cross-frontal exchange processes in the Gulf Stream based on isopycnal RAFOS float data. J. Phys. Oceanogr. 19, 1177-1190.

Bower, A.S., Lozier, M.S., 1994. A closer look at particle exchange in the Gulf Stream. J. Phys. Oceanogr. 24, 1399-1418.

Bower, A.S., Rossby, H.T., Lillibridge, J.L., 1985. The Gulf Stream: barrier or blender? J. Phys. Oceanogr. 15, 24-32.

Cronin, M., Watts, D.R., 1996. Eddy mean flow interaction in the Gulf Stream at $68^{\circ}$ W. J. Phys. Oceanogr. 26, 2107-2131.

del-Castillo-Negrete, D., Morrison, P.J., 1993. Chaotic transport by Rossby waves in shear flow. Phys. Fluids A 5 (4), 948-965.

Duan, J.Q., Wiggins, S., 1996. Fluid exchange across a meandering jet with quasi-periodic time variability. J. Phys. Oceanogr. 26, 1176-1188.

Dutkiewicz, S., Griffa, A., Olson, D.B., 1993. Particle diffusion in a meandering jet. J. Geophys. Res. 98 (C9), $16487-16500$.

Flierl, G.R., Malanotte-Rizzoli, P., Zabusky, N.J., 1987. Non-linear waves and coherent vortex structures in barotropic $\beta$-plane jet. J. Phys. Oceanogr. 17, 1408-1438.

Haller, G., Poje, A.C., 1998. Finite time transport in aperiodic flows. Physica D 119, 352-380.

Hogg, N.G., 1988. Stochastic wave radiation by the Gulf Stream. J. Phys. Oceanogr. 18, 1687-1701.

Lacasce, J.H., Bower, A.S., Zhang, H, 2000. Relative dispersion in the subsurface North Atlantic. J. Mar. Res., submitted for publication.

Lozier, M.S., Bercovici, D., 1992. Particle exchange in an unstable jet. J. Phys. Oceanogr. 22, 1506-1516.

Malhotra, N., Mezić, I., Wiggins, S., 1998. Patchiness: a new diagnostic for Lagrangian trajectory analysis in time-dependent fluid flows. Int. J. Bifurcation Chaos Appl. Sci. Eng. 8, 1053-1093.

Malhotra, N., Wiggins, S., 1998. Geometric structures, lobe dynamics, and Lagrangian transport in flows with aperiodic time-dependence, with applications to Rossby wave flow. J. Non-linear Sci. 8, 401-456.

Meyers, S.D., 1994. Cross-frontal mixing in a meandering jet. J. Phys. Oceanogr. 24, 1641-1646.

Miller, P.D., Jones, C.K.R.T., Rogerson, A.M., Pratt, L.J., 1997. Quantifying transport in numerically generated vector fields. Physica D 110, 105-122.

Ngan, K., Shepherd, T.G., 1997. Chaotic mixing and transport in Rossby wave critical layers. J. Fluid Mech. 334, 315-351.

Pedlosky, J., 1987. Geophysical Fluid Dynamics, (2nd edition). Springer. 710 pp.

Poje, A.C., Haller, G., Mezić, I., 1999. The geometry and statistics of mixing in aperiodic flows. Phys. Fluids 11, 2963-2968.

Pratt, L.J., Lozier, M.S., Beliakova, N., 1995. Parcel trajectories in quasi-geostrophic jets: neutral nodes. J. Phys. Oceanogr. 25, 1451-1466.

Rogerson, A.M., Miller, P.D., Pratt, L.J., Jones, C.K.R.T., 1999. Lagrangian motion and fluid exchange in a barotropic meandering jet. J. Phys. Oceanogr. 29, 2635-2655.

Samelson, R.M., 1992. Fluid exchange across a meandering jet. J. Phys. Oceanogr. 22, 431-440.

Savidge, D.K., Bane, J.M., 1999a. Cyclogenesis in the deep ocean beneath the Gulf Stream. 1. Description. J. Geophys. Res. 104 (C8), 18111-18126.

Savidge, D.K., Bane, J.M., 1999b. Cyclogenesis in the deep ocean beneath the Gulf Stream. 2. Dynamics. J. Geophys. Res. 104 (C8), 18127-18140.

Spall, M., 1992. Cooling spirals and recirculation in the subtropical gyre. J. Phys. Oceanogr. 22, 564-571.

Wiggins, S., 1992. Chaotic Transport in Dynamical Systems. Interdisciplinary Applied Mathematics, Vol. 2. Springer, Berlin, 301 pp. 